\title{
La formation d'un espace public camerounais à l'épreuve de la construction des réseaux routiers de communication
}

\author{
Joseph Keutcheu*
}

\begin{abstract}
Résumé
La question centrale de notre communication est donc la suivante : peut-on construire et consolider un espace public au Cameroun sans une intégration physique de son territoire, sans les réseaux de communications ? La réponse à cette question nous amènera à envisager ces réseaux de communication comme vecteurs de l'imposition de l'ordre étatique sur le territoire et, simultanément, comme le moyen de mise en réseau d'acteurs d'une société civile très souvent contemptrice de la puissance publique. Ce faisant, à travers la l'inégale distribution spatiale des réseaux de communication et la révélation d'une société en archipel, on met en exergue la configuration baroque de l'espace public camerounais.
\end{abstract}

\begin{abstract}
The central issue of this article is as follows: Can a public space be built and consolidated in Cameroon without the physical integration of its territory, without communications networks? In answering this question, we envisage communications networks as vectors for imposing government control on the territory and, simultaneously, as a way of forming a network of civil society actors that often denigrate the public authorities. In so doing, through the uneven spatial distribution of communication networks and the revelation of an archipelago society, we highlight the baroque configuration of Cameroonian public space.
\end{abstract}

* Université de Yaoundé II, Cameroon. E-mail : jkeutcheu@yahoo.fr 


\section{Introduction}

L'élaboration, par Jürgen Habermas, du concept d'Öffentlichkeit ou espace public à la fin des années 50 et au début des années 60 a constitué une avancée théorique considérable dont on n'a pas fini de tirer les enseignements. Pour autant, on ne peut nier, d'une part, que ce concept a été en quelque sorte victime de son succès et, d'autre part, qu'il n'est pas exempt de défauts. Il est frappant, en effet, de constater que la formule « espace public » est souvent utilisée à mauvais escient ou, en tout cas, qu'elle est employée dans une acception tellement large qu'elle n'a plus qu'un très lointain rapport avec la théorie d'Habermas ${ }^{1}$. Il est donc logique de dire ce que l'espace public veut dire avant de dire l'espace public dans son rapport au réseau routier. Pour Habermas, l'espace public est ce lieu de discussions formelles et informelles situé, selon une topographie mouvante et incertaine, quelque part entre la société civile et l'Etat. Il constitue une sorte de « caisse de résonance » susceptible de "répercuter les problèmes qui, ne trouvant de solution nulle part ailleurs, doivent être traités par le système politique » (Habermas 1997:386). Nous le considérons simplement comme le lieu d'identification et de thématisation des pans problématiques du monde vécu, comme un réseau permettant de communiquer des contenus et des prises de position, et donc des opinions sur les questions de société. C'est un espace symbolique où s'opposent et se répondent les discours, la plupart contradictoires, tenus par les différents acteurs politiques, sociaux, religieux, culturels, intellectuels, composant une société. Aussi la modeste ambition de cette présente contribution est-elle d'établir un rapport entre construction du réseau routier et la perspective génétique d'un espace public au Cameroun. Le projet tient évidemment compte de ce que le réseau routier est un élément parmi tant d'autres que l'on peut prendre en compte dans une telle perspective. On fait notamment allusion ici à la turgescence du discours sur les réseaux numériques portés au rang de deus ex machina de l'intégration sociale (Rheingold 1993 ; Coisne et Soussin 1998; Castells 2001; Granjon 2001).

Aborder les infrastructures de transport et de circulation notamment le réseau routier, comme objets d'étude en sciences sociales en général et en science politique en particulier peut sembler curieux, compte tenu du statut scientifique dudit objet. En effet,

Dans la cité des chercheurs, les réseaux techniques [notamment routiers] restent encore rejetés dans les bas-fonds de la connaissance véritable impensés urbanistiques. [Ils] ne semblent mériter qu'un regard condescendant, $[\ldots]$ la chose technique motive peu les sciences sociales (Offner 2000:137). 
Bien peu de choses ont été écrites sur les réseaux routiers, notamment sur leurs incidences sociopolitiques, culturelles et surtout territoriales. On rappellera dès lors que ce relatif vide scientifique semble concerner bien d'autres réseaux en Afrique qui apparaissent comme les parents pauvres d'une science politique jalouse de son pré carré : processus électoral, ethnicité, élites politiques, institutions politiques. La modeste ambition du présent papier est, entre autres, de s'intéresser à ces objets délaissés que sont les routes dans leur configuration en réseau ${ }^{2}$, d'investir des terrains de recherche encore insuffisamment explorés. Il s'agit en fait d'intégrer dans la production réflexive les choses les moins pensées (Bourdieu 2002; Peretti-Watel 2002), car les plus communément admises. L'intérêt d'une pensée en termes de réseau et non en termes de segments, de tronçons tient à la prise en compte du rôle social et territorial des routes, c'est-à-dire au fait qu'ils relient différents lieux, différents territoires, différents individus. Le réseau routier est ainsi posé comme analyseur social et politique dans la mise en ordre de l'espace public. La question initiale du présent papier est donc la suivante : dans quelle mesure la construction de réseaux de communication tels que les réseaux routiers participe-t-elle à la formation d'un espace public camerounais?

Interroger le rapport entre voies de circulation et formation de l'espace public nous met en demeure de mettre en exergue le public dans l'espace public. Comprendre le public dans l'espace public, c'est interroger sa relation avec l'espace, ses modes d'usage et de consommation, voire ses types d'appropriation : c'est interroger la consommation "mécanique " de l'espace, d'une part, (usage simple) et l'usage «humain » qui remonte à des enjeux politiques, sociaux, spatiaux, psychologiques, symboliques, historiques et culturels, d'autre part (relation complexe) (Tisseron 1999:61). Comprendre le public dans l'espace public, c'est interroger sa relation et son rôle dans la constitution et dans la conception même de l'espace qu'il s'approprie : c'est interroger sa participation comme acteur principal qui a une grande connaissance de son espace. Dans la présente communication, la pratique du réseau routier constitue la clé principale de compréhension du public dans l'espace public, étant entendu que l'espace public renvoie, dans une perspective habermasienne, à un espace de débat politique, qui concourt à la formation de l'opinion et de la volonté des citoyens et permet l'élaboration d'une critique des pouvoirs et des institutions en place ainsi que l'expression de nouveaux besoins, transmis depuis la périphérie du système politique jusqu'à son centre. On note donc un lien intrinsèque entre construction du réseau routier camerounais et construction de réseaux sociaux, d'une société en réseau. 


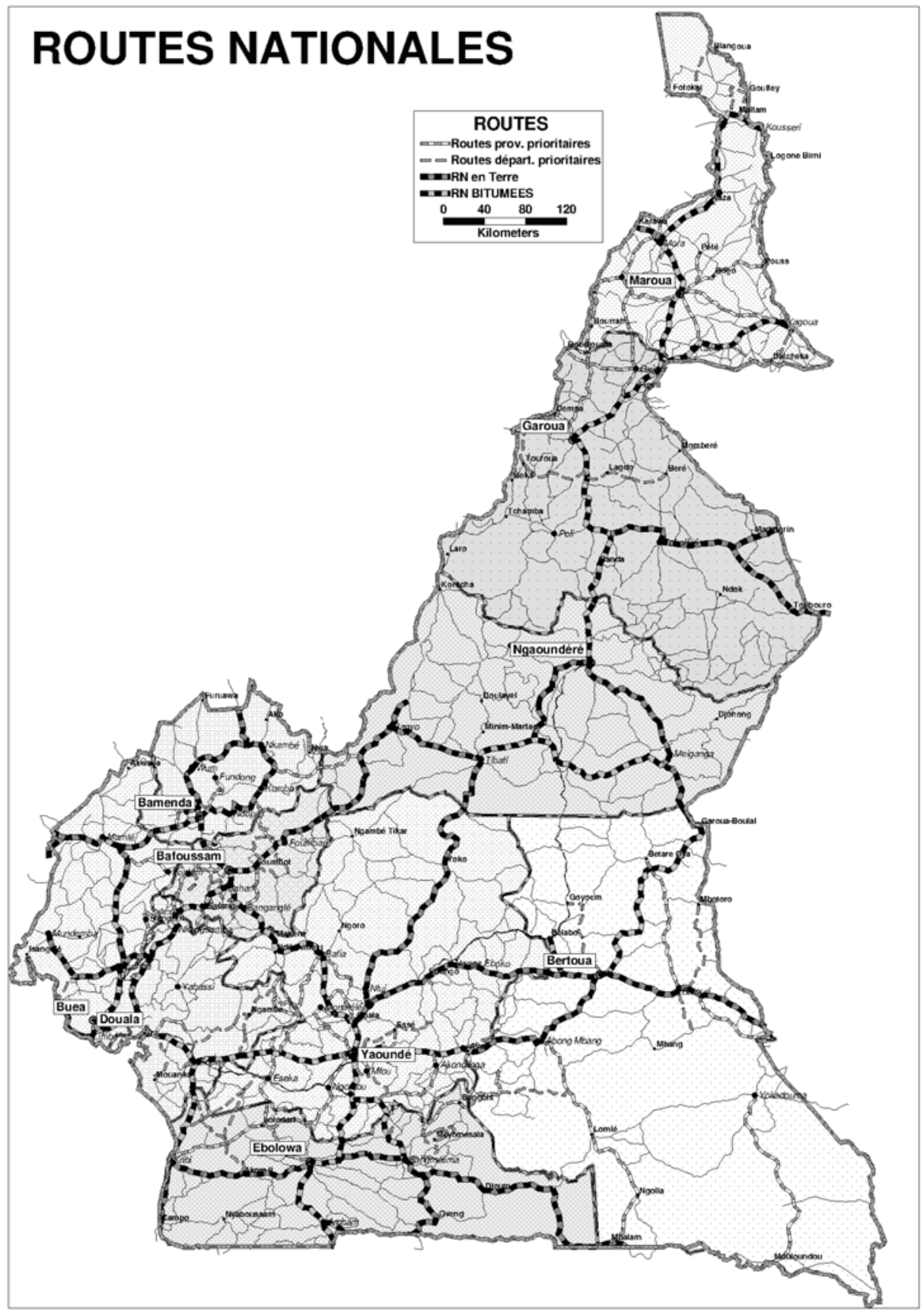

Institut national de la cartographie, Yaoundé, 2009 
Nous voulons de ce fait indiquer que l'analyse de la formation d'un espace public au Cameroun et même les discussions relatives à la viabilité d'un tel espace ne peuvent faire l'économie d'un arrêt sur l'implantation de ces infrastructures phares et, donc, sur la mise en relation des Camerounais, sur l'activation subséquente d'un processus interactif complexe (I). On replace ainsi la notion « d'espace public » là où elle émerge, c'est-à-dire à l'intersection des champs de l'aménagement du territoire et des sciences sociales (Betin 2001:47-54) ${ }^{3}$. Ce faisant, à travers l'inégale distribution spatiale des réseaux de communication et la révélation d'une société en archipel, on met en exergue la configuration baroque de l'espace public camerounais (II).

\section{Le projet de formation d'un espace public camerounais peut-il faire l'économie de la construction des voies de circulation?}

Espace physiquement inscrit dans le sol, un espace immatériel où circulent les idées, l'espace public camerounais est le produit des réseaux de l'intersubjectivité. Les réseaux de communication, notamment les réseaux routiers, lient inter-subjectivement les Camerounais au sens où ceux-ci habilitent l'individu à vaincre son isolement et à devenir un acteur proprement social. La formation d'un espace public camerounais est, dans une grande mesure, redevable au génie du territoire qui se manifeste à partir de l'implantation d'infrastructures de communication telles que les routes sur un espace géographique, propriété de l'Etat (2). Ce sont en effet les infrastructures qui donnent sens à l'espace, qui forment les territoires et qui permettent d'en mobiliser les ressources. Elles sont à la fois la trace et le moyen de toutes les grandes réalisations politiques. Dès lors, l'enclavement, perçu comme un mal, qu'il s'agisse du processus de fermeture causé par un obstacle originel ou plutôt par une accumulation d'obstacles spatialement concentrés, est envisagé comme obstacle à la projection de l'ordre politique et à la construction d'un espace public.

L'enclavement : subversion du principe de publicité et rédhibitoire à l'éclosion d'un espace public camerounais

L'espace public est abordé ici dans la perspective habermassienne, sans préjudice des différentes critiques afférentes à cette perspective ${ }^{4}$, comme le lieu où s'élabore la critique des pouvoirs, des institutions, des injustices, où se formulent des revendications identitaires, sociales, politiques ou culturelles, où se forment par des voies à la fois formelles et informelles un pouvoir communicationnel susceptible d'influencer les pouvoirs judiciaire et administratif. Le processus de création dudit espace public au Cameroun est concomitant à celui de la formation de l'Etat. En effet, dans la pratique, il existe un rapport fort entre le processus de territorialisation, c'est-à-dire 
l'établissement progressif d'un ordre politique sur le territoire camerounais et l'élaboration, l'opérationnalisation du principe de publicité qui est au centre de la dynamique de l'Etat. Dans cette optique, la territorialisation désigne le fait de

Construire et reconstruire sans cesse ce qui environne l'acteur social, matériellement et dans ses représentations : pour l'institution, c'est son aire de pouvoir ou d'influence ; pour l'individu, c'est une subite « alchimie» entre du personnel et du collectif (Di Méo 1996).

La construction du monolithe politique Cameroun résulte de la systématisation progressive de ses grands ensembles sociogéographiques (voir figure 1). Sans faire dans la « politologie de la carte postale ${ }^{5}$ », il convient de noter que le contrôle de l'espace et sa structuration par le pouvoir central est fortement dépendant de la « domestication » des aspérités physiques de l'espace. Penser la construction de l'Etat par un détour spatial passe en effet par la prise en compte de ces considérations topographiques. Il s'agit, notamment pour le pouvoir central, de neutraliser les freins à la circulation que constitue le relief accidenté de l'ouest et, ainsi, de capter la périphérie occidentale de l'Etat jusqu'aux confins de la frontière nigériane. L'impératif est de prendre pied au pays anglophone et de réussir une fois pour toutes l'intégration des territoires anglophone et francophone. Autrement dit, il faut traduire géographiquement et concrètement la Réunification réalisée en octobre 1961. C'est dans cette perspective qu'il faut ranger la construction de l'axe routier Yaoundé-Bafoussam-Bamenda, un axe pleinement inscrit dans le schéma fonctionnel de l'intégration du territoire camerounais (voir figure $\mathrm{n}^{\circ} 2$ ).

Dans un tel contexte de territorialisation, les voies de communication telles que les routes se révèlent être les instruments d'une cybernétique spatialisée au sein de l'Etat, mais aussi des instruments de mise en contact de l'individu avec son environnement immédiat et étendu. Elles participent à l'opérationnalisation du principe de publicité, donc au processus de construction de sens, le sens de l'Etat sur un espace territorialisé et labellisé Cameroun. Elles participent au processus d'imposition de d' « esprits d'Etat » sur ledit espace (Bourdieu 1993:49-62). De fait, la construction des routes participe de la mise en place de structures d'encadrement politique des populations camerounaises et, ce faisant, " produit du territoire ", selon l'expression d'Alliès (1980). C'est notamment à travers la route que le sens de l'Etat, maître d'ouvrage, se construit dans des localités telles que Mokolo, Mora et Kousseri à l'extrême-nord, Tibati, Tignère, Meiganga et Toubouro dans l'Adamaoua, Belabo, Garoua-Boulaï et Batouri à l'est, Nkambé, Kumbo et Wum dans le nord-ouest (voir carte). 
Figure 1 : Le Cameroun éclaté ou l'appel à commutation spatiale

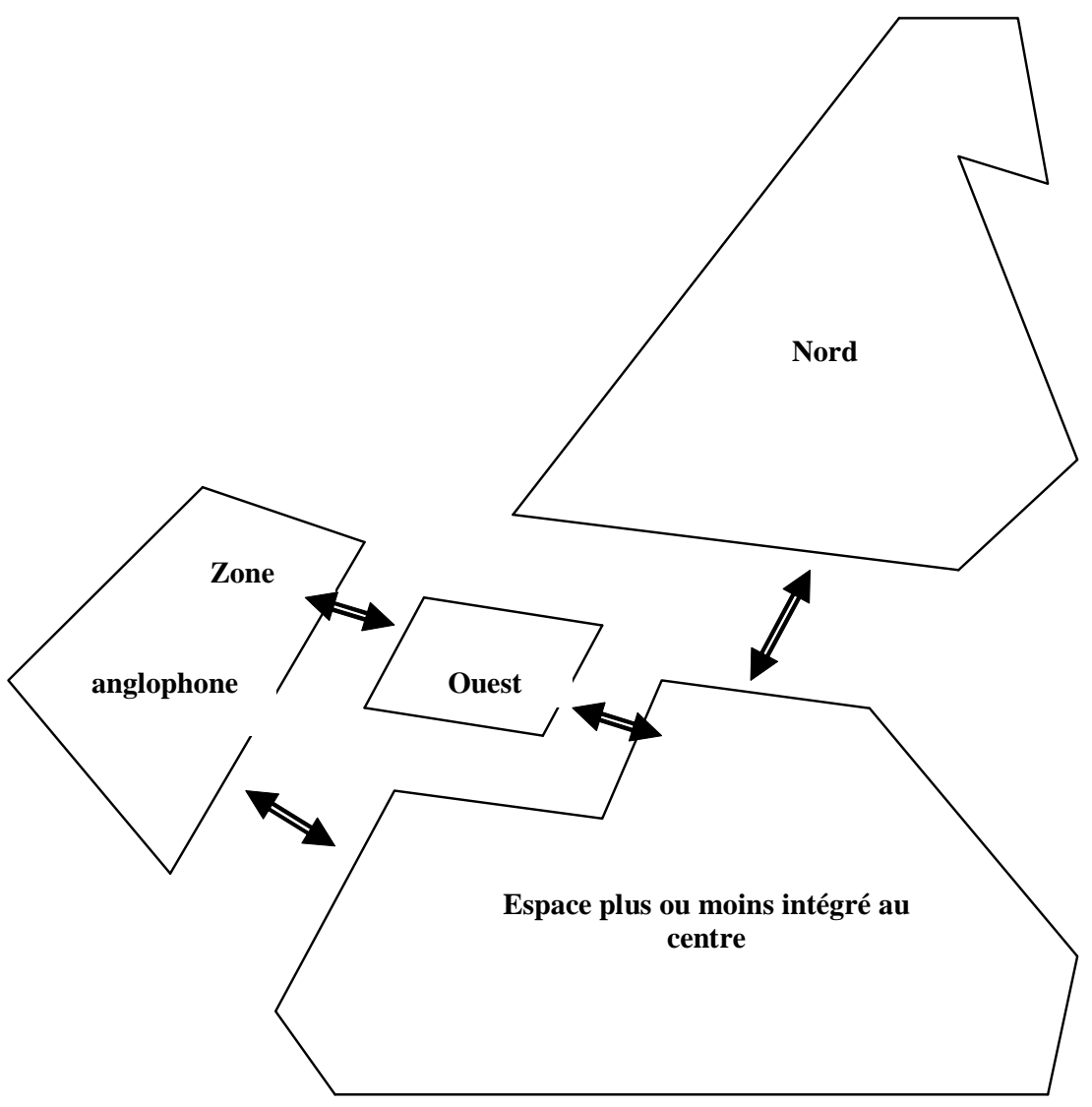

$\square$ Espace territorialisé ou en territorialisation

$\Longrightarrow \quad$ Axe de la territorialisation 
Fondement de la théorie habermassienne de l'espace public, le principe de publicité constitue à nos yeux un concept qui porte loin et qui, mutatis mutandis, reste une référence pour analyser les rapports entre l'Etat camerounais et la société. Le principe de publicité constitue un des éléments essentiels de l'Etat, en même temps qu'un des principes fondamentaux du droit. Au Cameroun, les conséquences pratiques qui en attestent sont extrêmement nombreuses. D’une façon générale, la validité des lois, règlements, actes administratifs, jugements, délibérations des collectivités territoriales, etc., est subordonnée à leur publication et/ou à leur notification aux intéressés à peine de nullité. Dans un contexte où l'écoute de la radio et de la télévision se développe essentiellement dans le milieu urbain et continue à être rare en zone rurale, la route est le vecteur premier de cette publication, c'est le support des supports. A titre d'illustration, cela signifie concrètement que tout mariage entraîne la publication des bans, que les lois et décrets doivent impérativement être publiés au Journal Officiel, que les délibérations d'un conseil municipal doivent obligatoirement être affichées à l'extérieur de la mairie, que les jugements ne sont exécutoires qu'après avoir été rendus publics et notifiés aux condamnés par lettre recommandée, etc. Sauf exception prévue par la loi, tout ce qui concerne l'Etat, le gouvernement, le Parlement, les organes délibérants des collectivités territoriales, est nécessairement public, non seulement pour ce qui est des décisions, mais aussi pour ce qui est des délibérations: ainsi, toutes les assemblées élues, du conseil municipal à l'Assemblée nationale, se réunissent en séance publique, les procès sont publics, et il faut des circonstances très particulières, exceptionnelles, pour qu'intervienne le huis clos. Ainsi, dans le principe et dans la pratique, cet espace public favorise la co-présence des différentes communautés sur le sol camerounais. Envisagé ainsi, l'espace public est antinomique de l'enclavement, cet épouvantail de l'aménagement du territoire de l'Etat.

L'enclavement se pose en s'opposant à la mouvance de la circulation et de l'ouverture à l'autre (Debrie et Steck 2001:27). C'est une situation qui prend le contre-pied de l'opérationnalisation du principe de publicité et donc de l'émergence d'un espace public camerounais. La notion renvoie ainsi à une situation de fermeture spatiale. L'enclavement s'inscrit dans le champ lexical de l'emprisonnement, du blocage. Dès lors, considérer les localités de Ngoila et de Garigombo dans la province de l'Est, d'Akwaya dans la province du Sud-Ouest comme des zones enclavées revient à considérer qu'elles sont en situation d'isolement, de blocage, d'enfermement par rapport au « centre » politique yaoundéen et aux autres localités. C'est le fait de l'isolement géographique et métrique qui constitue ces localités en « enclaves ». En effet, ces zones sont étymologiquement des enclaves, car elles sont partiellement fermées ou isolées dans un système de relations. Cet 
enclavement constitue de ce fait un rédhibitoire à l'intégration de ces localités à l'espace public camerounais. Cette situation empêche en effet ou limite l'Etat dans son ambition de rendre publics, de faire connaître au public de ces localités, ses actes, ses décisions, ses projets, ses délibérations, etc., soit directement, soit par l'intermédiaire de la presse, de façon à ce que les citoyens, dûment informés, puissent organiser un débat public dans lequel ils puissent faire publiquement usage de leur Raison, afin que puisse se constituer une opinion publique éclairée, la Loi et l'action de l'Etat étant censés refléter ladite opinion publique.

Le concept d'enclavement est alors en rapport avec l'écart entre les projets de mobilités et les possibilités réelles d’ouverture (Debrie 2005:4). Le plus souvent, cette approche est réticulo-centrique, c’est-à-dire basée sur l'idée de réseaux, notamment de réseaux de communication ; l'absence d’infrastructures de transport est alors perçue comme isolement, marginalisation, enclavement. Dès lors, considérer les localités de Ngoila et de Garigombo dans la province de l'Est, d'Akwaya dans la province du Sud-Ouest comme des zones enclavées revient à considérer qu'elles sont en situation d'isolement par rapport au « centre » politique yaoundéen du fait de leur inaccessibilité, du fait de l'absence de réseaux fiables de communication permettant de les relier au lieu-centre ou aux centres-relais. Ce sont donc des localités en situation de marginalisation, étant entendu que la marginalisation dans ce contexte est envisagée comme une contrainte négative, comme un rédhibitoire aux actions d’intégration politique et de production d'un espace public camerounais (Pallier 1982:1190).

Mention doit cependant être faite de ce qu'il n'existe pas d'enclave, ni d'isolat absolu. A l'échelle du Cameroun, il n’existe pas de lieux totalement isolés des autres ; la quête irrésistible d'interactions dans les espaces contemporains est telle que les obstacles ne parviennent pas à créer de l'étanchéité entre ces lieux et les autres. Les fermetures sont le plus souvent partielles et relatives. C'est dans cette optique que Jean Debrie n’hésite pas à dire que « la problématique de l'enclavement-désenclavement renvoie bien à la position des lieux sur le gradient qui va de la fermeture à l'ouverture » (Debrie 2005:1). L'enclavement des localités de Ngoila et de Garigombo dans la province de l'Est, d'Akwaya dans la province du Sud-Ouest n'est pas intégral, car il y existe des flux en direction de centres-relais. Il se trouve simplement que sur le gradient qui va de la fermeture à l'ouverture, ces localités connaissent des situations proches de la fermeture. Il y a également lieu de dire que l'enclavement peut être contingent et conjoncturel. A titre d'illustration, à l'occasion de l'effondrement, le 2 juillet 2004, du pont sur le Mungo, les localités telles que Tiko, Limbé, Buea, jusque-là ouvertes au reste du pays, se sont momentanément retrouvées en situation d'enclavement relatif, 
car elles ne pouvaient plus être facilement en contact avec une grande partie du pays, y compris avec le centre yaoundéen. Dans le même ordre d'idées, la figure de l'enclavement relatif et contingent est prédicable aux zones qui se trouvent en situation de fermeture par rapport à certains centres-relais ou au centre yaoundéen du fait du coût élevé du transport. En empruntant le langage économique, on peut dire que la hausse des tarifs de transport a eu un effet immédiat sur l'utilité marginale à voyager ou à se déplacer d'une grande partie de la population camerounaise dont les revenus sont modestes. Si ces coûts sont prohibitifs, le transfert d'une zone A à une zone B ne peut plus se faire. L'intégration de telles localités à l'espace public camerounais s'en trouve donc affectée. La présence ou l'absence de voies de circulation est ainsi au cœur du procès d'une sphère publique camerounaise.

\section{Voies de circulation : sociogenèse et procès de la sphère publique camerounaise}

Nous abordons les réseaux de communication comme des instruments de projets de continuité, de mise en relation et d'ouverture. On peut dire avec Nicolas Currien que ce sont des outils "d'intermédiation et d'interconnexion » (Currien 1992). Dans cette optique, la formation de l'espace public camerounais est fortement justiciable de la mise en place de réseaux de communication et du processus occurrent de création d'une opinion publique nationale (Blondiaux 1998). Elle est tributaire de la production de flux d'informations et des interconnexions entre les composantes sociopolitiques du Cameroun (Degenne et Forsé 2004:35-56 ; Chevallier 1985:79-81). Dans cette perspective, les routes au Cameroun permettent une configuration sociétale du pays. En effet, toute action communicative a pour but l'intersubjectivité. Les infrastructures routières plongent les individus dans les réseaux et permettent la force de l'intersubjectivité socialisée. Il est question, à partir de cette posture, de déconstruire les fausses évidences de la pratique quotidienne des routes qui, très souvent dans le langage commun, sont confinées au simple statut d'instruments de transport des biens économiques. D'ailleurs, tout le débat à propos de la construction des routes au Cameroun et toute la glose sur l'enclavement de la région $\mathrm{X}$ et du désenclavement de la région Y sont fortement structurés par cet impensé économique. Sortir de l'usage routinier des routes dans le champ discursif revient à prendre au sérieux les interconnexions et interdépendances induites par ces infrastructures comme productrices d'un espace public.

Il y a lieu de noter que l'espace public qui naît ainsi à travers le réseau routier est co-produit dans le cadre de la formation de l'Etat camerounais (voir figure). Il est le produit du processus de territorialisation et d'intégration mené par l'Etat en même temps qu'il procède de la dynamique des usagers 
Figure 2 : Schéma fonctionnel de l'intégration du territoire camerounais
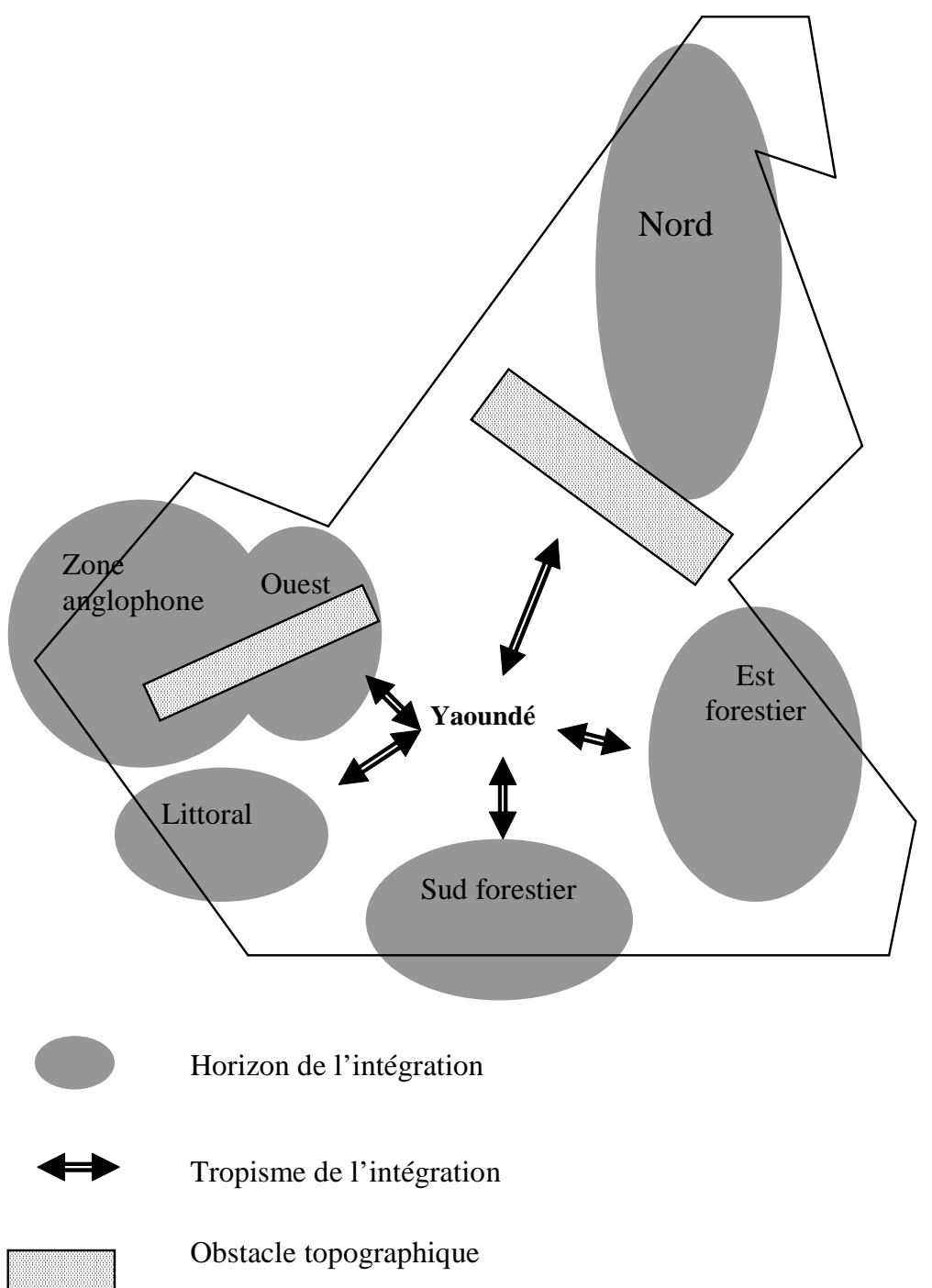

KEUTCHEU Joseph 
du réseau routier qui disposent à l'égard de cet objet technique d'une autonomie créatrice qui leur permet de développer des tactiques d'appropriation ou éventuellement de résistance capables d'agir sur la forme de l'intégration envisagée (Lambert 2004:113). On peut donc penser qu'en tant qu'espace de rencontre, espace d'expression sociopolitique, «l'espace public, c'est sans doute l'espace qui se dessine, qui se conçoit et qui se programme, mais c'est aussi l'espace qui ne se décrète pas, et qui se co-produit dans le vécu et dans les perceptions de ses usagers » (Levasseur 1991:9).

Les voies de circulation modernes comme instruments de monopolisation de l'espace politique au Cameroun, mais aussi comme levier de production de l'espace public camerounais, s'inscrivent originellement dans un champ chronologique précis, les périodes successives de domination allemande, française et britannique, puis la période postcoloniale. Dans cette perspective, nous estimons que pour intelliger l'espace public en formation, il faut être en mesure de retracer le processus de construction des " complexes institutionnels » relevés par Antony Giddens (1990), processus qui, au Cameroun, est inséparablement lié à la mise en place des infrastructures de communication. En effet, selon Giddens, l'agrégation progressive des « complexes institutionnels » est au cœur de la naissance de l'Etat. Il s’agit notamment du rapprochement surveillé des populations dispersées sur un espace en territorialisation, rapprochement qui est constitutif d'un marché politique et économique national ; de l'appropriation des fonctions contraignantes et représentatives qui lui confère une assise territoriale et de l'accumulation du pouvoir administratif qui améliore les modes de gouvernance. Evidemment, cette agrégation a fortement partie liée avec la tutelle progressive sur la distance. On comprend donc que la construction des voies de communication soit au principe de la production d'une infrastructure territoriale nationale et d'un Etat au Cameroun. Elle est dans le même temps au principe de l'émergence d'un espace public camerounais, car à travers les flux d'informations et de personnes qu'elle facilite, elle participe à la constitution d'une opinion publique camerounaise.

Dans la perspective de la co-production ci-dessus évoquée, il y a lieu de relever qu'en même temps que le réseau routier qui se met progressivement en place au Cameroun pendant la période coloniale favorise la territorialisation des dominations allemande, française et anglaise, il participe à la territorialisation du sentiment anti-colonial. On est ainsi en plein dans « le malentendu colonial » (Keutcheu 2006:27-38). L'imperium des puissances allemande, française et anglaise au Cameroun repose grandement sur le recours à la construction des infrastructures de contrôle politique telles que les voies de communication. Ce contrôle politique des hommes se fait évidemment par la médiation du sol ; le territoire devient un instrument de 
l'action politique (Badie 1995:12). Ici, l'enjeu est précisément l'occupant du territoire : la population. Il est question de la mettre au service de l'exploitation de la rente coloniale. Paradoxalement, cette technologie de contrôle des populations et du territoire camerounais sert également à diffuser la subversion de l'autorité coloniale. A titre d'illustration, c'est à travers le processus de territorialisation (par la construction des voies de communication) qu'ils initient que les Allemands parviennent à fédérer contre eux les mécontentements des populations.

Dès lors, on peut difficilement confiner le sentiment anti-colonial dans une partie du pays; le maillage colonial du territoire par les voies de communication telles que les routes suppose évidemment la dissémination des méthodes d'administration récriminées et donc la dissémination du sentiment anti-colonial. A titre d'illustration, la carte du maillage routier du Cameroun allemand correspond pratiquement à celle de la répartition de la subversion de l'ordre politique germanique au Cameroun : révolte de Lock Priso de Hickory Town (Bonabéri) et de Elamé de Joss (Douala) en 1884; des Bakweri en 1894, de Lionn de Ngila en 1895, des Ewondo conduits par Omgba Bisogo de 1895 à 1896, des Bene conduits par Amougou Baana de 1897 à 1898, des Boulou sous la conduite de Oba’a Mbeti (1899-1900), des Bafut et des Bandeng en 1901, du Lamido Soulé de Rey Bouba en 1901, du Lamido Oumarou de Banyo en 1902, de l'émir de Yola en 1902, des chefs Bangwa à l'ouest du pays en 1903, etc. (Owona 1996:90-91 ; Ebune 1992:24). En charriant l'identité nationale camerounaise, le réseau routier colonial participe en fait à l'émergence d'un espace public camerounais, un espace social très informel qui ne requiert, de la part de ceux qui y participent, que la maîtrise d'une langue naturelle et la capacité élémentaire de prendre part aux pratiques quotidiennes de la communication. On voit clairement à l'œuvre le « génie du lieu », c’est-à-dire, « [...] ce qui fait que tant bien que mal, sur un territoire donné, les uns s'ajustent aux autres, et les uns et les autres à l'environnement naturel [et politique]» (Maffesoli 1988:35).

Quid de la période postcoloniale ? Elle correspond simplement à un moment de recyclage de l'espace public camerounais né pendant la période coloniale. Ici aussi, le réseau routier mis en place s'inscrit dans les logiques spatiales de la construction de l'Etat. Dans l'étude du rapport entre route et formation d'un espace public, trois fonctions du réseau routier camerounais sont repérées : une fonction d'usage, une fonction de message, une fonction d'ordre. En ce qui concerne l'usage, on envisage le réseau routier comme permissif de la circulation. La route, tout en érodant la distance entre les lieux et les groupes humains, favorise la mobilité des personnes, mais aussi et surtout la diffusion de l'information à travers le territoire de l'Etat. A côté de cette simple facilitation de la circulation des hommes et des messages, se 
Figure 3 : Les nodalités politiques ou points de projection de l'Etat

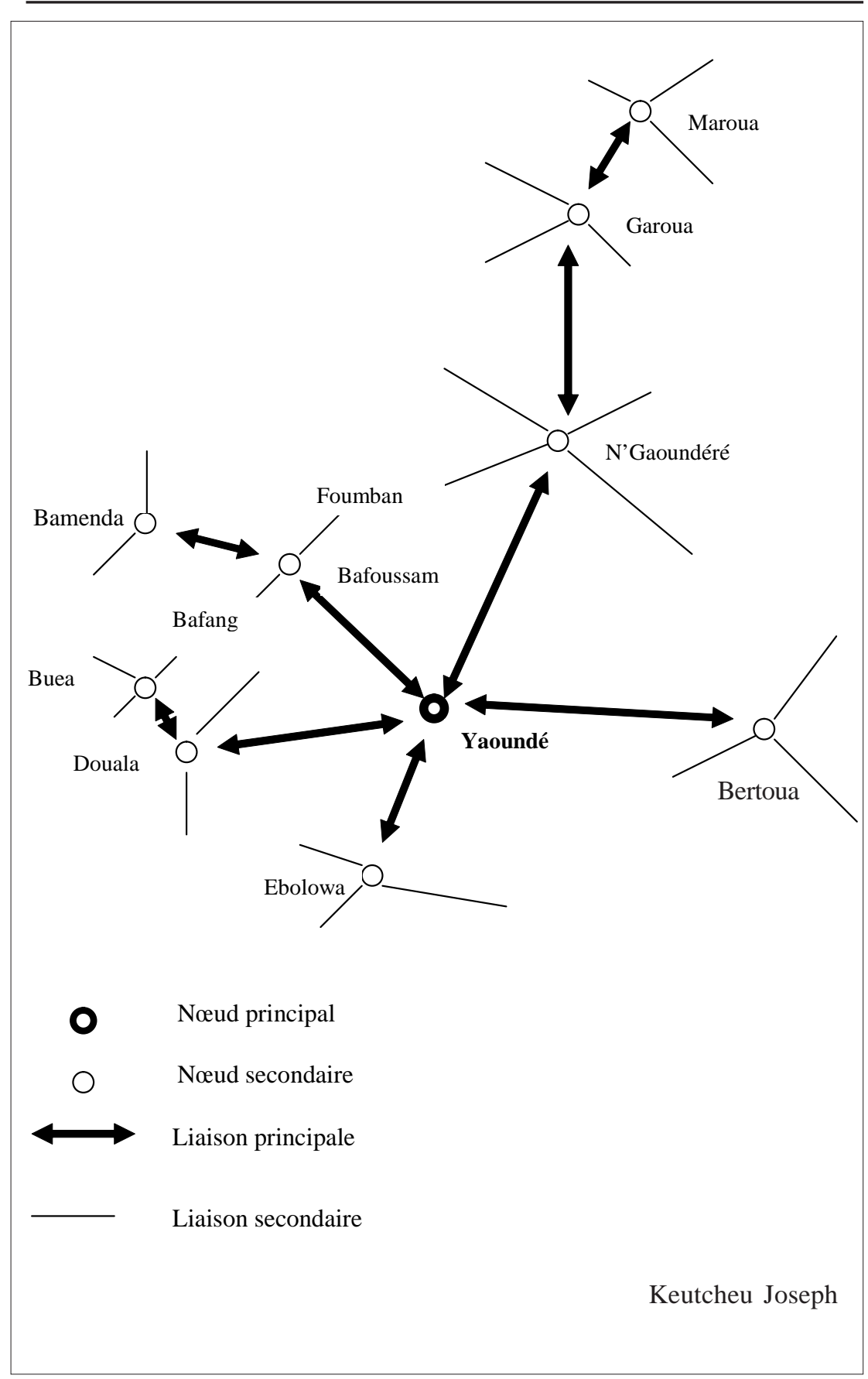


joue une véritable opération politique menée par l’Etat postcolonial: le déploiement du processus d'établissement de l'imperium sur le territoire. Autrement dit, la construction des voies de communication permet la diffusion d'un ordre politique dans un espace approprié par l'Etat. Ces trois fonctions opèrent autant sur le registre de l'apparent que sur celui du caché. Point n’est besoin ici de désolidariser usage, message, ordre ; l'apparent d'une fonction fait diversion pour entretenir le caché d'une autre fonction. Tout compte fait, le réseau routier est au cœur des situations d'interactions qui font la trame du lien social.

Ainsi, on ne devrait pas considérer comme quantité négligeable la dimension performative des routes dans l'affiliation de ces populations. Le réseau routier participe à l’homogénéisation de l'hétérogénéité engagée par l'Etat postcolonial. Les routes apportent de la « valeur ajoutée » à la vision d'une identité nationale, elles renforcent le discours politique qui y est relatif. A titre d'illustration, l'axe routier Yaoundé-Bafoussam-Bamenda permet le contact et même le rapprochement physique puis symbolique des ethnies traversées : Ewondo, Eton, Manguissa, Yambassa, Bamiléké, etc. Il en va de même de l'axe Douala-Yaoundé qui permet d'articuler Ewondo, Bassa, Bakoko, Duala, etc. En ce sens, la route est à l'origine de la naissance d'un « territoire circulatoire », c’est-à-dire un territoire qui permet un marché multidimensionnel entre les ethnies qui y sont présentes (Tarrius 1995:32). Théorisant la nation building, Deutsch (1966) relève l'importance des communications de masse qui intensifient les interactions entre ressortissants d’un territoire et permettent à la population de s’homogénéiser. En effet, les voies de communication en général et les routes en particulier, si l'on s'en tient au modèle de l'appareil circulatoire, permettent une socialisation commune de ces populations. Autrement dit, elles participent à l'acquisition d'une culture étatique commune. En l'occurrence, il s'agit d'une culture qui se caractérise par un tropisme unificateur que véhicule d'ailleurs abondamment le discours politique. Ainsi, comme pendant la période coloniale, la construction des réseaux routiers allait de pair avec l'ambition de subordination des volontés. La mise en place d'infrastructures de communication constitue une autre modalité de la diffusion capillaire de l'autorité étatique sur le territoire.

\section{Le rapport voies de circulation/formation de l'espace public camerounais à l'épreuve de la pratique quotidienne}

L'espace camerounais qu'étudient les géographes et les politistes a cessé d'être naturel. Il est structuré par des réseaux de communication, notamment le réseau routier. La mise en place de ceux-ci coûte cher, si bien qu'une 
opposition se dessine entre les zones équipées (on pourrait dire « branchées ») et celles qui ne le sont pas, ce qui ne va pas sans affecter la configuration de l'espace public camerounais.

\section{Du territoire "archipel " ou l'espace public en pointillés}

Le concept de réseau social est sous-jacent à l'étude des modes de sociabilité introduits par la construction des réseaux routiers au Cameroun. Il peut être défini comme une configuration de relations sociales (Coenen-Huther 1993:33). La notion de réseau au sens de configuration de relations sociales, est au cœur de la formation de l'espace public camerounais. Norbert Elias apparaît sans aucun doute comme le pionnier de la conceptualisation de la société comme réseau. Dans l'un de ses ouvrages de référence, il propose l'idée que la société est un conglomérat d'ensembles d'individus en interaction qui forment des configurations de relations sur une base de l'interdépendance réciproque (Elias 1991:156). Or, précisément, les voies de communication sont à la base de la nouaison de la société en réseau au Cameroun. Toutefois, l'observation de la carte routière dressée par le Ministère des travaux publics (MINTP) de ce pays laisse apparaître une inégale répartition de ces voies de circulation et même de leur qualité, ce qui introduirait de fait des continuités et des discontinuités dans la chaîne d'interactions sociales et donc dans l'espace public.

Dans certaines régions du pays, ces réseaux de communication ne remplissent que très imparfaitement leur fonction de liaison. C'est peu de dire que les usagers souffrent des conditions de circulation éprouvantes dans les provinces de l'Est, de l'Adamaoua, du Nord et de l'Extrême-nord. Il s'agit évidemment d'une énumération non exhaustive qui ne rend pas justice aux habitants d'autres localités qui ne souffrent pas moins de l'inexistence ou de l'état désastreux des routes. Selon le Plan directeur routier du Cameroun (MINTP 2006), " les routes sont en général dans un état mauvais ou médiocre ; sur le secteur prioritaire, seulement 12 pour cent de routes bitumées et 5 pour cent de routes en terre sont en bon état ", les autres réseaux (non prioritaire et rural) étant presque entièrement en mauvais état. Le tableau de la composition du réseau routier camerounais le montre d'ailleurs à suffisance. On pourrait arguer a contrario que les réseaux de télécom-munication, notamment à travers le prosélytisme téléphonique ambiant, ignorent les contraintes de la circulation au sol et pourraient pallier ces insuffisances du réseau routier ; toujours est-il que ces infrastructures de télécomunication ne peuvent se substituer aux nécessaires échanges terrestres. 
Composition du réseau routier camerounais (en $\mathbf{k m}$ )

\begin{tabular}{lrrc}
\hline Classe & Bitumé & Terre & Total \\
\hline Nationales & 3344 & 3853 & 7197 \\
Provinciales & 835 & 5109 & 5943 \\
Départementales & 652 & 7506 & 8158 \\
Rurales (non classées) & & 28300 & 28300 \\
Total & $\mathbf{4 8 3 0}$ & $\mathbf{4 4 6 7 8}$ & $\mathbf{4 9 5 9 8}$ \\
\hline
\end{tabular}

Source : MINTP, Plan directeur routier, 2006

Il ressort de ce tableau le constat de la qualité désastreuse du réseau routier camerounais, qualité qui déteint forcément sur les flux des hommes et des idées sur le territoire et donc sur la mobilisation politique ${ }^{6}$ et la formation d'un espace public (Deutsch 1966:126-128). En tout cas, il n'y a que quelques localités du pays qui bénéficient de routes en bon état. Si on introduit la difficulté à circuler sur les routes non bitumées en saison de pluies, on comprend que le transport routier souffre de l'état des routes et du surplus de la demande par rapport à l'offre. Ainsi, sur un total de 49598 kilomètres de routes, seulement 4830 kilomètres sont bitumés. La densité routière totale (linéaire de routes sur superficie du pays) est seulement de l'ordre de $4 \mathrm{~km} / 100 \mathrm{~km}^{2}$. Cela est d'autant plus marquant qu'en dépit de la mise sur pied du Programme sectoriel des transports (PST) au Cameroun, le financement pérenne et adéquat de l'entretien routier est loin de donner satisfaction (MINTP 2006).

La catégorie de l' « archipel » nous semble donc pertinente pour l'analyse de la formation de l'espace public camerounais, avec toutes les conséquences que l'on peut en tirer en termes de participation politique et de démocratie. La notion d'archipel évoque des phénomènes qui s'inscrivent dans quelques espaces restreints. Par exemple, dans l'étude de l'économie mondialisée actuelle, des géographes comme Pierre Veltz parlent d'une « économie d'archipel » : l'économie mondiale est contrôlée à partir d'un nombre restreint de métropoles bien reliées entre elles par des moyens de communication performants, des voies maritimes aux télécommunications. Ces métropoles apparaissent comme des îles isolées sur une mer. Il en va de même dans le contexte du Cameroun où on note en effet l'existence d'une juxtaposition d' « îles de communication », c'est-à-dire de régions relativement dotées en voies de communication, par conséquent, des régions marquées par d'importants flux de personnes, d'idées et de biens, à côté d'autres régions 
relativement peu dotées en infrastructures de même types, et qui se retrouvent pratiquement coupées du reste du pays. Il en découlerait une configuration baroque de l'espace public camerounais du fait de cette « archipélisation » de facto de l'Etat. La carte routière du Cameroun laisse apparaître que la cohésion du système géopolitique camerounais, fondée sur un réseau de circulation en grande partie routier, est très fragile. On y voit en exergue les parties septentrionale et méridionale, deux monolithes structurels à l'intérieur desquels les disparités ne laissent pas d'intriguer.

La superficie de près de $475000 \mathrm{~km}^{2}$ est insuffisamment et inégalement couverte. Le constat de la multiplication récente de routes nationales structurantes dans la partie méridionale du pays ne parvient pas à effacer l'idée de l'inégalité dans ladite partie. La densité routière par province est l'une des entrées permettant de relever cet état des choses. Le tableau cidessous donne les différentes densités au kilomètre carré par province dans la partie méridionale du Cameroun.

\section{Densité routière au $\mathrm{Km}^{2}$ au Cameroun méridional}

\begin{tabular}{lccc}
\hline Provinces & Superficie $\left(\mathrm{en} \mathrm{km}^{2}\right)$ & \multicolumn{2}{c}{ Total } \\
& & Linéaire & Densité $\left(\mathrm{km} / 100 \mathrm{~km}^{2}\right)$ \\
\hline Centre & 69000 & 11036 & 15,99 \\
Est & 110000 & 4974 & 4,52 \\
Littoral & 20000 & 2979 & 14,90 \\
Nord-Ouest & 16000 & 4504 & 28,15 \\
Ouest & 13000 & 4391 & 33,78 \\
Sud & 47000 & 4501 & 9,58 \\
Sud-Ouest & 25000 & 2991 & 11,96 \\
Cameroun & 464000 & 49802 & 10,73 \\
\hline
\end{tabular}

Source : d'après les données de la Direction des routes au MINTP.

De ce tableau, il ressort le constat de ce que les provinces de l'Est et du Sud ont des densités routières au-dessous de la moyenne nationale. Cela s'explique en grande partie par la présence dans ces provinces d'un important massif forestier protégé, la réserve du Dja, mais aussi par la faible densité de la population et l'insuffisance des investissements dans ce secteur des travaux publics. 
Trois « îles de communication » existent de fait dans la partie méridionale du Cameroun et constituent en quelque sorte les « anneaux utiles » de l'espace public camerounais (voir figure 3) :

- La première, à configuration étoilée, a pour centre Bafoussam. Véritable bouillon de culture démographique et, par conséquent, point de départ de vagues migratoires incessantes, ce centre étend ses ramifications, direction nord-est vers Foumbot et Foumban, direction nord-ouest vers Bamenda, direction sud-ouest vers Bafang, Nkongsamba et Douala, direction sud-est vers Bangangté et Yaoundé. La mobilisation politique relativement forte ici peut être envisagée comme un processus induit, entre autres, de la multiplication des voies de circulation et de la mise en réseau des populations.

- La deuxième île a pour point focal la ville de Douala qui est l'un des points de départ de l'arbre généalogique de l'urbanisation du Cameroun et la porte d'entrée et de sortie non seulement du pays, mais aussi de l'Afrique centrale. La ville concentre aussi les principales activités économiques du pays et forme avec ses alentours, notamment Edéa, Mbanga, Penja, Tiko, une véritable conurbation en gestation. Autant de caractéristiques qui en font un important centre-relais de la mobilisation politique au Cameroun et l'un des creusets de l'espace public camerounais. Les principales routes sont orientées ouest, vers Buéa et les grandes plantations des alentours, nord, vers Nkongsamba et Bafoussam, est, vers Edéa et Yaoundé.

- La troisième île de communication, un des principaux pôles de la formation de l'espace public camerounais est la ville de Yaoundé et son environnement immédiat. C'est le centre d'impulsion politique du pays, le siège des principales institutions de l'Etat et le point de départ du contrôle social et de la totalisation de l'espace géographique camerounais par l'Etat (Sindjoun 2002:35-38; Badie 1980:81-99).

Cette partie méridionale du Cameroun est fortement marquée par le tropisme occidental de ses voies de circulation et de ses activités. L'impression laissée à un observateur attentif de cette configuration est que le tropisme est si fort qu'il semble avoir dépourvu les ailes est et sud-est de tout dynamisme ; les voies de communication y existent de manière résiduelle et la mobilisation politique y est faible, de même que l'espace public. Ces parties apparaissent donc de fait comme les « anneaux inutiles » de l'espace public camerounais.

La partie septentrionale du pays, quant à elle, est structurée par l'axe méridien Ngaoundéré-Garoua-Maroua-Kousseri. Ici, si l'on prend en compte la mobilisation politique et la formation d'un espace public à travers les 
voies de communication, deux goulots d'étranglement peuvent être relevés. Le premier obstacle à surmonter est celui de la distance auquel sont liées les conditions géomorphologiques difficiles, le massif de l'Adamaoua constituant une frontière naturelle et difficilement surmontable entre les parties septentrionale et méridionale du pays 7 . Elle est susceptible de mettre en échec la relation entre pouvoir politique et espace, relation à l'origine du processus de territorialisation, c'est-à-dire du double mouvement d'appropriation matériel et idéel permanente dudit espace par l'Etat. On comprend dès lors l'acharnement des politiques publiques dans la lutte contre la distance et dans la promotion de la proximité. Pour l'Etat, le problème de l'espace peut être vu comme la gestion des contradictions engendrées par la distance, qui empêche l'interaction entre le centre et sa périphérie. Les politiques d'aménagement du territoire, notamment la construction des voies de communication, apparaissent alors comme des moyens de gestion de ces contradictions.

La distance entre le centre politique camerounais et la partie septentrionale du pays n'a pu être encore domestiquée de manière optimale. On est encore loin de la proximité recherchée, proximité qui est au cœur de la construction d'un « faire ensemble », d'un « dire ensemble » donc, au cœur de la formation d'un espace public camerounais. La construction et la polarisation des singularités entre "nordistes » et « sudistes » au Cameroun ont ainsi fortement partie liée à la distance géographique entre les localités d'origine des deux groupes qui ne sont pas facilement en interaction. La plupart de nos interlocuteurs lors de nos séjours de recherche dans la province de l'extrême-Nord manipulait de manière récurrente le " vous là-bas à Yaoundé » ou "les gens du Sud », expressions marquant une distanciation nette entre eux et les acteurs politiques réels ou supposés du « centre ». En effet, on peut relever la minimisation du temps de trajet, de sa longueur, de son coût, etc. comme des indicateurs de la proximité entre deux lieux, entre deux acteurs ou groupes d'acteurs qui entreprennent de se connecter. Une distance se mesure, pour une organisation comme pour les individus, en minutes ou en heures mises pour entrer en contact avec le partenaire. De ce point de vue, elle s'apparente dans une large mesure à la distance coût de transport (Rallet 2002).

La distance rend difficile la mobilité entre le nord et le sud et donc la mise en relation permanente et massive entre les populations des deux entités. Dupuy et Torre considèrent que « la proximité géographique peut constituer, par l'établissement de relations répétées d'une culture commune et de liens de coopération, voire de confiance, un facteur de cohésion (...) favorable à l'établissement et à la pérennisation de relations de coopération » (2000:77). 
Dès lors, l'effet de la distance agit sur l'ajustement de l'offre et de la demande de mobilité en fonction du coût de déplacement. La distance n'agit que si elle est proportionnelle au coût de mise en relation. Les acteurs obéissent en effet au principe du moindre effort. De toute évidence, la rationalité économique est accolée à la question du rapport entre distance et mobilité.

Le deuxième goulot d'étranglement est la quasi inexistence de bras de pénétration ou de communication secondaires ; l’axe méridien NgaoundéréGaroua-Maroua-Kousseri est très peu articulé à des voies horizontales véritablement viables, si l’on exclut Garoua-Yagoua et le récent NgaoundéréToubouro. Cela participe à la constitution de vastes pans du territoire camerounais en isolats dont le tropisme est davantage extérieur. Cela accrédite l'idée selon laquelle le territoire camerounais, loin de former un tout cohérent, est un amalgame de micro-territoires qui, dans la pratique, semblent s’autonomiser. On note de ce fait un écartèlement de la partie septentrionale du Cameroun en deux mouvances tournées vers l’extérieur :

- La première mouvance est orientale et tournée prioritairement vers le Tchad. Il est constant que de Toubouro dans l'Adamaoua jusqu'à Goulfey dans l'extrême-Nord, un large pan du Cameroun subit, faute de mieux, l'attraction du Tchad.

- La deuxième mouvance, occidentale, est davantage tournée vers le Nigéria. De Mogodé et même au-delà dans l'extrême-Nord jusqu’à Ribao dans l’Adamaoua, un chapelet de localités camerounaises vit plus au rythme de la conjoncture socio-économique du Nigeria.

Ainsi se présente la formation de l'espace public camerounais au moment où s’affirme le besoin d'un nouveau cadre de réflexion pour l'avenir. Il s’agit d'une réflexion qui prend nécessairement en compte la question de la gouvernance territoriale.

L'articulation du réseau routier à la gouvernance territoriale : les douze travaux d'Hercule

Le problème de l'implantation des réseaux de communication soulève de nombreuses questions de gouvernance ${ }^{8}$. La gouvernance convoquée ici met l'accent sur la crise de la gouvernabilité du territoire camerounais du fait de son écartèlement structurel, du fait de son « archipélisation » à l'aune des voies de communication. Il s'agit d'un concept qui rend compte des transformations des formes de l'action publique. Le premier des problèmes est celui de la participation politique. En effet, la communication pèse sur l'espace public. Il est difficile, dans ce cas, d'envisager la mobilisation politique des habitants des zones enclavées et leur participation à l'espace public, il est difficile de voir de tels individus inscrits dans la série de normes sociales qui 
président le fonctionnement d'un tel espace. Ainsi, pendant que les populations des « îles de communication » s'ouvrent de plus en plus et jouissent de plus en plus d'une participation politique multiforme (vote, marches de protestation, grèves, etc.), celles des zones enclavées en sont privées ou du moins n'en bénéficient que de manière épisodique et non optimale (lors des élections par exemple). On peut faire une analogie entre la restriction, telle que décrite par Habermas, de l'espace public européen du $18^{\mathrm{e}}$ siècle à l'élite capable de s'acquitter du cens et le contexte camerounais actuel. L'accessibilité ici est devenue une espèce de cens moderne qui permet aux habitants de certaines régions d'avoir plus voix au chapitre que d'autres. Il est d'ailleurs symptomatique que les agitations politiques de la fin des années 1990 ayant entraîné la mise en crise de la culture autoritaire au Cameroun ${ }^{9}$

Il ne peut y avoir participation si on ne se sent pas partie prenante d'un groupe, sans sentiment d'appartenance, sans disposer de moyens de se faire entendre - moyens institutionnels certes, mais aussi des ressources personnelles. La dépendance matérielle, le défaut de savoir, le sentiment d'incompétence, une situation d'exclusion produisent l'apathie. Une situation d'exclusion telle que la vivent les populations de zones enclavées peut aussi nourrir des réactions de rejet et/ou de repliement sur des identités particulières et différentialistes. La montée de ce qu'il est convenu d'appeler l'exclusion se trouve au cœur de la crise de la participation politique au Cameroun, sans en être, bien sûr, toute l'explication. On se trouve ainsi en plein dans le casse-tête du rapport entre participation politique et démocratie (Mayer et Perrineau 1992:15-18; Perrineau 1994:40-50). L’idéal démocratique repose en effet sur la conception que le pouvoir politique procède du peuple souverain. Il ne peut donc y avoir de démocratie sans implication du citoyen, c'est-à-dire sans citoyen actif. C'est pourquoi la citoyenneté politique postule non seulement la jouissance de droits civiques attachés à la nationalité (le droit de vote, l'éligibilité, les libertés publiques, l'accès aux fonctions de responsabilité), mais aussi le devoir de s'impliquer dans la vie politique, d'y participer au moyen, entre autres, d'une inscription dans les réseaux de communication (Brechon 2003:64-68). La question se pose donc comme un défi à des pouvoirs publics camerounais engagés dans le processus de démocratisation de la société : au lieu d'activer principalement les leviers institutionnels de la démocratie, n’y a-t-il pas lieu de prendre aussi sérieusement en compte la question de la gouvernance territoriale à travers notamment la question des voies de communication?

C'est la participation véritable du citoyen à la chose publique qui est questionnée dans l'analyse du rapport entre réseaux routiers et formation de l'espace public camerounais ; c'est aussi la question de la gouvernance territoriale qui est soulevée. De fait, la question soulevée permettrait de faire 
un saut qualitatif de l'idéal de la participation politique au Cameroun à l'épreuve des faits. La mise en place des infrastructures de circulation telles que les routes ouvrent véritablement le territoire camerounais aux flux de communication de tous genres, du centre vers la périphérie et inversement. La construction des voies de communication, en inscrivant les Camerounais dans des réseaux de communication, concourait à la fabrication du « citoyen ordinaire » participant au développement territorial et aux choix politiques qui engagent la nation. La mise en place d'une démocratie participative est en forte prise avec la question de la construction des voies de communication. La gouvernance est ainsi systématiquement utilisée comme un terme permettant d'identifier de nouvelles pratiques collectives, de nouvelles formes d'actions publiques qui ne reposeraient plus seulement sur la domination et la violence légitime, chères à Max Weber, mais sur la négociation, le partenariat : la gouvernance par opposition au gouvernement, on n'impose plus, on négocie. Dans ces approches, la gouvernance sert à désigner l'ensemble des pratiques collectives par le biais desquelles l'ordre social et politique est produit et qui prennent place en dehors des institutions classiques.

Le deuxième défi de gouvernance qui découle de l’ « archipélisation » de l'espace public camerounais est celui de l'équité territoriale. En d'autres termes, la question du lien entre réseaux routiers et territoire, entre réseaux routiers et espace public se pose sous l'angle de l'équité. Certes, l'équité est d'abord une notion d'éthique qui a marqué l'effort réflexif des philosophes (Emmanuel Kant dans La métaphysique des møurs, 1797; Jean-Jacques Rousseau dans Le contrat social, 1835 ; John Rawls dans Theory of Justice, 1971). Mais elle traduit l'aspiration à une relative égalité de traitement entre les citoyens, entre les habitants d'une même région, aspiration dont se saisissent les politiques publiques (Derycke et Offner 1997:5-7). Loin de notre propos l'ambition d'embrayer sur les considérations d'ordre éthique ; il est question plutôt de l'examen des politiques publiques de l'éthique en matière de voies de communication. Concrètement, l'équité territoriale ici sous-entend la notion de «service universel » que se doivent de remplir les autorités publiques dans la dotation du pays en voies de communication. La carte routière du Cameroun révèle cependant une distribution inégale des voies de communication qui induit une hiérarchisation de fait d'espaces dans un même territoire. Les localités des « îles de communication » bénéficient davantage des externalités positives du réseau routier que celles des zones enclavées (coût des transports, localisation des entreprises, emplois, ...). Il en découle inexorablement une dynamique démographique différenciée du fait de l'attraction des premières sur les habitants des secondes, ce qui ne rend pas justice à l'équité dans la distribution spatiale de la croissance économique. 
En réalité, les espaces les plus dynamiques sont logiquement situés à proximité des pôles de communication ou des grands axes de communication. Douala et Yaoundé et leur périphérie immédiate, qui ont connu une évolution significative de leur population totale, semblent avoir bénéficié de l'apport d'habitants issus des bassins démographiques périphériques. Il s'agit d'un constat qui remet au goût du jour l'aménagement du territoire, notamment du territoire de ces deux métropoles. Les problèmes actuels d'occupation anarchique de l'espace, les solutions imaginées qui passent par le déguerpissement forcé des occupants des zones inconstructibles, les débats occurrents participent, dans une certaine mesure, à la décrédibilisation des actions de l'Etat en matière d'aménagement du territoire. Une des solutions structurelles sur laquelle les pouvoirs publics insistent, mais pas encore suffisamment, est celle de l'équité territoriale, la dotation équitable du territoire camerounais en infrastructures.

La question du rapport entre réseau routier et formation d'un espace public camerounais peut aussi être abordée à partir de la lucarne de l'économie politique. C'est à ce niveau que se situe le troisième défi posé par l' " archipélisation » de l'espace public au Cameroun. Le faible niveau général des infrastructures routières et leur inégale distribution spatiale sont des entraves à une intégration géoéconomique nationale dynamique sur laquelle les pouvoirs publics comptent dans l'optique de la construction nationale (Guilaumont 1985:344 ; Grellet 1986:127). La lecture du paysage économique camerounais donne à constater une trop grande concentration des activités (banques, industries, assurances, services commerciaux,...) à Douala et à Yaoundé. Les raisons de la rentabilité économique expliquent en partie un tel déséquilibre spatial dans la répartition des activités. Mais cela n’explique pas tout. L'inégale distribution des voies de communication est l'une des causes de ces distorsions. Or, à côté des équipements traditionnels de développement, le potentiel des routes pourrait contribuer à une meilleure intégration des territoires et des activités économiques.

\section{Conclusion}

Au terme de cette réflexion sur le rapport entre construction du réseau routier et formation de l'espace public camerounais, force est de retenir que la communication de masse surdétermine l'espace public camerounais. Dans un contexte d'appropriation progressive mais encore insuffisante des nouvelles technologies de l'information et de la communication, la route conserve toute sa pertinence dans l'émergence d'une sphère publique au Cameroun. Rendant visibles le politique et la société, participant à la diffusion des informations, les réseaux de communication qui naissent du réseau routier participent incontestablement de l'espace public. Elle participe à la 
vulgarisation de la norme étatique et la publicisation des décisions alimente les consciences individuelles.

Il s'agit cependant d'un statut qui est fortement érodé par une situation de fait : le développement inégal du réseau de voies de communication sur le territoire camerounais entraîne une « insularisation » dudit territoire, avec des zones fortement intégrées qui coexistent avec de véritables isolats. Il en découle un Cameroun configuré en «Etat archipel». Il s'agit sans doute d'une situation qui n'est pas singulière au Cameroun : Pourtier, Bruneau et Simon avaient déjà évoqué un cas quasi similaire au Zaïre (Pourtier 1991; Bruneau et Simon 1991). La notion d'espace public camerounais en archipel nous invite fondamentalement à réfléchir sur les rapports entre routes, communication et démocratie. En effet, au fond, l'espace public repose sur l'idée d'universalité, une norme qui le voudrait accessible à tout citoyen. Ceci place au premier plan la configuration du réseau routier camerounais. Si le réseau routier constitue le trait majeur de l'espace public camerounais, il s'ensuit normativement qu'il devrait rester techniquement et économiquement à la portée des membres de la société. L'exclusion d'un segment quelconque de la population du fait de l'enclavement entre en contradiction avec la prétention de la démocratie camerounaise à l'universalisme. L'idée de l'espace public camerounais que nous avons développée se fonde sur la recherche de quelque chose de commun, de partagé, auquel tous les citoyens ont droit. La mise en place d'un espace public camerounais est sans doute en marche. Reste à se prémunir de la subversion du principe de publicité que le phénomène d'enclavement participe à entretenir.

Toutefois, on doit dire au terme de ce papier que l'espace public ne consiste pas simplement en la mise en place de réseaux de communication à travers les routes, puisqu'il implique l'engagement des citoyens dans les affaires publiques, ainsi que dans leurs actions réciproques. La communication entre citoyens est un élément décisif de l'espace public, mais une telle communication dépend des conditions nécessaires, sociales et structurelles. Les réseaux de communication sont nécessaires comme catalyseurs et ressources, mais la qualité de l'espace public dépend de la participation des citoyens.

Au total, au moment où ont lieu des tentatives pour construire l'espace public dans les Etats africains, nous devons garder à l'esprit que cela ne constitue pas pour autant une garantie de la démocratie ; c'est certainement nécessaire, mais pas suffisant. Si la notion d'espace public souligne les dimensions communicatives et interactives des démocraties, l'attention en Afrique doit également être dirigée vers les aspects traditionnels des structures politiques, les processus décisionnels, l'engagement civique et les aspects pratiques du pouvoir. 


\section{Bibliographie}

Alliès, P., 1980, L'invention du territoire, Grenoble, Presses universitaires de Grenoble.

Badie, Bertrand, 1980, Le développement politique, Paris, Economica.

Betin, Christophe, 2001, « La construction de l'espace public. Le cas de Lyon», Géocarrefour, vol. 76, n 1, pp. 47 - 54.

Blondiaux, Loïc, 1998, La fabrique de l'opinion, Paris, Seuil.

Bourdieu, Pierre, 2002, Le bal des célibataires. Crise de la société paysanne en Béarn, Paris, Seuil.

Bourdieu Pierre, 1993, «Esprits d'Etat. Genèse et structure du champ bureaucratique ", Actes de recherche en sciences sociales, $\mathrm{n}^{\text {os }}$ 96-97, mars 1993, pp. 49-62.

Brechon, P., 2003, « La participation politique : crise et/ou renouvellement », Cahiers Français, n ${ }^{\circ}$ 16, septembre - octobre 2003, pp. 64-74.

Bruneau, Jean-Claude et Thierry, Simon, 1991, «Zaïre, l'espace écartelé », Mappemonde, $4^{\mathrm{e}}$ trimestre 1991, pp. 1-5.

Castells, Manuel, 2001, La Galaxie Internet, Paris, Fayard.

Chevallier, Jacques, 1985, Eléments d'analyse politique, Paris, PUF.

Coenen-Huther, Jacques, 1993, « Analyse de réseaux et sociologie générale », Flux, vol. 9, n 13, pp. $33-40$.

Coisne, E. et Soussin, F., 1998, Internet@visages humains, Éditions d'Organisation.

Debrie, Jean, 2005, « L’enclavement : expression géographique de la discontinuité dans les réseaux », communication au 16 ${ }^{\mathrm{e}}$ Festival International de Géographie, Saint-Diè-des-Vosges, du 29 septembre au 2 octobre 2005, [en ligne], disponible sur : http://fig-st-die.education.fr/actes/actes_2005/debrie/ debrieenclavement.pdf., référence du 02 juillet 2007.

Debrie, Jean et Steck, Benjamin, 2001, « L’enclavement, réévaluation théorique et application à l'Afrique de l'Ouest », Espace géographique, n 1, pp. 26-36.

Degenne, Alain et Forsé, Michel, 2004, Les réseaux sociaux, Paris, Armand Colin.

Derycke, Pierre-Henri et Offner, Jean-Marc, 1997, « Réseaux et équité territoriale : présentation », Flux, ns 27/28, janvier-juin 1997, pp. 5-7.

Deutsch, Karl, 1966, Nationalism and Social Communication, Cambridge, MIT Press.

Di Méo, George, 1996, Les territoires du quotidien, Paris, L’Harmattan.

Ebune, Joseph B., 1992, The growth of political parties in Southern Cameroons. 1916-1960, Yaoundé, CEPER.

Elias, Norbert, 1991, Qu'est-ce que la sociologie ?, Paris, Agora.

Giddens, Anthony, 1990, The consequences of modernity, Cambridge, Polity Press.

Granjon, F., 2001, L'Internet militant, Éditions Apogée.

Grellet, G., 1986, Structures et stratégies du développement économique, Paris, Presses Universitaires de France.

Guilaumont, P., 1985, Economie du développement. Tome 2. Dynamique interne du développement, Paris, Presses Universitaires de France. 
Habermas, Jürgen, 1997, Droit et démocratie, Paris, Gallimard.

Habermas, Jürgen, 1978, L'espace public, Paris, Payot

Lafargue, J., 1996, Contestations démocratiques, Paris, Karthala/IFRA

Lambert Stéphane, 2004, «Les télécommunications internationales et l'Etat occidental. Libertés de communiquer et relations internationales », thèse de doctorat de science politique, Institut d'études politiques de Paris.

Levasseur, J., 1991, « L'espace public », in Isaac J. (dir.), L'espace du public, les compétences du citadin, actes du colloque d'Arc-et Senans du 8 au 10 novembre 1990 à Paris, Ed. Plan Urbain, collection recherches.

Maffesoli Michel, 1988, Le temps des tribus. Le déclin de l’individualisme dans les sociétés de masse, Paris, Méridiens Klincksieck.

Mayer, Nonna et Perrineau, Pascal, 1992, Les comportements politiques, Paris, Armand Colin.

Ministère des travaux publics, 2006, Plan directeur routier du Cameroun, Rome, AIC Progetti.

Offner, J-M., 2000, « Réseaux et dynamiques urbaines », in Paquot T. et al. La ville et l'urbain, l'état des savoirs, Paris, La découverte.

Owona Adalbert, 1996, La naissance du Cameroun. 1884-1914, Paris, L'Harmattan.

Pallier, G., 1982, Les problèmes de développement dans les pays intérieurs de l'Afrique occidentale, Thèse Doctorat d'Etat, Bordeaux III.

Peretti-Watel Patrick, 2002, « Sous les étoiles, rien de nouveau », Revue française de sociologie, 43-1.

Perrineau Pascal (dir.), 1994, L’engagement politique : déclin ou mutation ? Paris, Presses de Sciences-Po.

Pourtier Roland, 1991, "Zaïre. L’inéluctable défi des transports », Politique Africaine, ${ }^{\circ}$ 41, mars 1991, pp. 22-31.

Rallet, A., 2002, « L'économie de proximité. Propos d’étape », in Torre A., éd., Etudes et Recherches sur les Systèmes Agraires et le Développement, n³3, Le local à l'épreuve de l'économie spatiale, pp. 11-25.

Reingold, H., 1993, The Virtual community : Homesteading on the electronic frontier, MIT Press.

Sindjoun Luc, 2002, L'Etat ailleurs. Entre noyau dur et case vide, Paris, Economica.

Tarrius Alain, 1995, « Territoires circulatoires des entrepreneurs commerciaux maghrébins de Marseille : du commerce communautaire aux réseaux de l'économie souterraine mondiale », Journal des Anthropologues, n 59, pp. 15-35.

Tisseron Serge, 1999, « Nos objets quotidiens », Hermès, n 25, pp. 57-66. 
